\title{
The Association Between Fecal Short-Chain Fatty Acids, Gut Microbiota, and Visceral Fat in Monozygotic Twin Pairs
}

\author{
Xing-Qi Yin*, Ya-Xin An*, Cai-Guo Yu, Jing Ke, Dong Zhao, Ke Yu \\ Center for Endocrine Metabolism and Immune Diseases, Luhe Hospital, Capital Medical University, Beijing, People's Republic of China \\ *These authors contributed equally to this work \\ Correspondence: Ke Yu, Center for Endocrine Metabolism and Immune Diseases, Luhe Hospital, Capital Medical University, No. 82, Xinhua South \\ Road, Tongzhou District, Beijing, People's Republic of China, Tel +86 I38I I6576I8, Email yuke@ccmu.edu.cn
}

Purpose: To examine the association of short-chain fatty acids (SCFAs), gut microbiota and obesity in individual twins and to control for genetic and shared environmental effects by studying monozygotic intrapair differences.

Patients and Methods: The study recruited 20 pairs of monozygotic twins. Body composition measurements were performed by using the multi-frequency bioelectrical impedance technique. SCFAs were extracted from feces and quantified by gas chromatographymass spectrometer. Gut microbiota was evaluated by 16S rRNA gene sequencing.

Results: Fecal SCFAs were negatively correlated with adiposity parameters including body mass index, visceral adipose tissue and waist circumference (all $P<0.05$ ). Metastat analysis showed that the top 5 relatively abundant bacterial taxa of viscerally obese and non-obese groups were Bacteroides, Collinsella, Eubacterium rectale group, Lachnoclostridium, and Tyzzerella. Participants with visceral obesity had lower abundance of Bacteroides and Collinsella compared to non-obese patients $(P<0.05)$. Among them, the abundance of Collinsella was positively correlated with acetic acid concentrations $(r=0.63, P=0.011)$. There were no significant intrapair differences in each SCFA concentrations between the twins in our study $(P>0.05)$.

Conclusion: Low fecal concentrations of SCFAs were associated with visceral obesity, and the gut microbiota might be involved in the underlying mechanism.

Keywords: twins, short-chain fatty acids, gut microbiota, obesity, visceral fat

\section{Introduction}

In the last decades, the prevalence of obesity has increased worldwide. Obesity has significant impacts on health, psychosocial well-being, longevity, and quality of life. ${ }^{1,2}$ The human intestine contains approximately $10^{14}$ microorganisms, collectively known as the gut microbiota. ${ }^{3}$ Numerous physiological and clinical studies have demonstrated that gut microbiota plays a crucial role in the pathophysiology of metabolic diseases by regulating the energy intake, harvest, and substrate metabolism. ${ }^{4,5}$ Accumulated data have suggested that an ecologically dysfunctional gut microbiome is closely linked to the development of obesity. ${ }^{6}$ Short-chain fatty acids (SCFAs), consisting primarily of acetate, propionate, and butyrate, are produced through bacterial fermentation of the fibers. SCFAs provide an extra energy source equivalent to $10 \%$ of the daily caloric intake. However, in contrast to being extra energy source and adipogenic factors, SCFAs have been demonstrated to play a beneficial role in appetite regulation and lipid and glucose metabolism. ${ }^{7}$ So far, the role of SCFAs in host energy metabolism has not been fully clarified.

Monozygotic (MZ) twins, originating from one zygote and inherit nearly identical genetic material, naturally pair up and share a similar environment. ${ }^{8}$ Studies on twins intrapair differences are free from genetic and shared environment confounding, highlighting the individual differences caused by environmental influences not shared. ${ }^{9}$ Twins research began in the behavioral sciences and has expanded to several domains including nutrition and health. In recent years, 
researches arising from twins have begun to incorporate metabolomics analysis. ${ }^{10-13}$ The aim of this study is to examine the association of SCFAs, gut microbiota and obesity in individual twins, and to control for genetic and shared environmental effects by studying monozygotic intrapair differences.

\section{Materials and Methods Study Subjects}

Twenty pairs of MZ twins who grew up in the same family and resided in Tongzhou District of Beijing were finally enrolled in our study. The criteria for the inclusion of twins were aged 18-65, and free of heart disease, liver or renal disease, diabetes mellitus, neoplastic disease, uncontrolled thyroid disease, psychiatric or neurological disorders. They were excluded if they were pregnant, had reported drug/alcohol abuse, with a history of diarrhea or intestinal infection in the last 3 months before enrollment in the study. Twin volunteers were investigated in two ways. Firstly, the twins were divided into two groups according to the level of visceral adipose tissue (VAT) in each pair, and the intrapair differences in obesity-related indexes and SCFAs in MZ twins were studied. Secondly, all participants were considered as a whole and were divided into two groups based on VAT above $100 \mathrm{~cm}^{2}$ and VAT below $100 \mathrm{~cm}^{2}$ to investigate the association between obesity, gut microbiota, and SCFAs. Individuals with visceral fat exceeding $100 \mathrm{~cm}^{2}$ were defined as visceral obesity. The study was complied with the Helsinki Declaration for investigation of humans and it was approved by the Ethics Committee of Capital Medical University, China (2021-LHKY-059-02). All subjects provided informed consents.

\section{Clinical and Laboratory Measurements}

Physical examinations were performed including body weight, body height, waist and hip circumference. Body mass index (BMI) was calculated as weight divided by the square of height $\left(\mathrm{kg} / \mathrm{m}^{2}\right)$. VAT was measured by a multi-frequency bioelectrical impedance analyzer (Inbody 770, Biospace Co. Ltd.). Venous blood samples were collected in the morning following an overnight fast. An auto-biochemical analyzer (Roche/Hitachi Cobas C501, Roche Diagnostic Corp, Indianapolis) was employed to determine plasma concentrations of triglycerides (TG), total cholesterol (TC), low-density lipoprotein cholesterol (LDL-C), high-density lipoprotein cholesterol (HDL). Fasting blood glucose (FBG) and fasting insulin (FINS) were examined using electrochemiluminescent immunoassay on the E601 module of the Roche Cobas 6000 (Burgess Hill, West Sussex, U.K.). HOMA-IR was calculated using the following equation: HOMA-IR $=$ insulin $\times$ glucose/22.5. Glycosylated hemoglobin (HbA1c) concentrations were quantified using high-performance liquid chromatography (HPLC) with a D10 set (Bio-RAD, Hercules, California). Fecal samples were collected and stored frozen at $-80^{\circ} \mathrm{C}$ until further analysis and then sent to Beijing Genomics Institute to extract DNA and identify the zygosity.

\section{Quantification of Fecal SCFAs}

For the extraction and quantification of SCFAs, the samples were firstly thawed at room temperature, then those weighing $100 \mathrm{mg}$ were homogenized in $100 \mu \mathrm{L}$ of $15 \%$ phosphoric acid with $100 \mu \mathrm{L}$ of $250 \mu \mathrm{L} / \mathrm{mL}$ isohexanoic acid solution as IS and $400 \mu \mathrm{L}$ ether $(70 \mathrm{~Hz}$ for $1 \mathrm{~min})$. Subsequently, the samples were centrifuged at $4^{\circ} \mathrm{C}$ for 10 $\min (12,000 \mathrm{rpm})$ and the supernatant was transferred into the vial prior to gas chromatography-mass spectrometer (GC-MS) analysis. The GC was fitted with a capillary column Agilent HP-INNOWAX $(30 \mathrm{~m} \times 0.25 \mathrm{~mm}$ i.d. $\times$ $0.25 \mu \mathrm{m}$ ) (Agilent Technologies, Santa Clara, CA, USA) and helium was used as the carrier gas at $1 \mathrm{~mL} / \mathrm{min}$. The splitter ratio was $10: 1$, the injection volume was $1 \mathrm{ul}$, and the injector temperature was set at $250^{\circ} \mathrm{C}$. The temperature of the ion source, interface, and quadrupole were $230^{\circ} \mathrm{C}, 250^{\circ} \mathrm{C}$, and $250^{\circ} \mathrm{C}$, respectively. The column temperature was initially $90^{\circ} \mathrm{C}$, then increased to $120^{\circ} \mathrm{C}$ at $10^{\circ} \mathrm{C} / \mathrm{min}$, to $150^{\circ} \mathrm{C}$ at $5^{\circ} \mathrm{C} / \mathrm{min}$, and finally to $250^{\circ} \mathrm{C}$ at $25^{\circ} \mathrm{C} / \mathrm{min}$ and kept at this temperature for $2 \mathrm{~min}$ (total run-time of $15 \mathrm{~min}$ ). The detector was operated in electron impact ionization mode (electron energy $70 \mathrm{eV}$ ) using full scan and single ion monitoring (SIM) mode. The concentrations of acetic, propionic, butyric, isobutyric, valeric, isovaleric and caproic acids were determined using standard curves. 


\section{Microbiome Composition Analysis}

Total genome DNA from fecal samples was monitored by Qubit ${ }^{\circledR}$ dsDNA HS Assay Kit. V3 and V4 hypervariable regions of prokaryotic 16S rDNA were selected for amplification, classification analysis and sequence alignment. Quality control was carried out on a per-sample basis, discarding paired-ends with an overlap of less than 200nt and removing chimeric sequences using denovo chimera detection in USEARCH. The effective sequences were used in final analysis. Sequences were grouped into operational taxonomic units (OTUs) using the clustering program Vsearch software (Version 1.9.6) against the UNITE ITS database (https://unite.ut.ee/) pre-clustered at 97\% sequence identity. The Ribosomal Database Program (RDP) classifier was used to assign taxonomic categories to all OTUs at a confidence threshold of 0.8 .

$\alpha$ diversity and $\beta$ diversity of community were calculated with QIIME (Version 1.9.1) and displayed with R software (Version 2.15.3). $\alpha$ diversity metrics (Shannon index and Chaol index) were calculated on the rarefied OTU table using the alpha_rarefaction. $\beta$ diversity metrics (weighted and unweighted UniFrac distances) were calculated on the rarefied OTU table with the beta_diversity. Principal coordinate analysis (PCoA) is used to show the visualization of $\beta$ diversity.

\section{Statistical Analysis}

The data were analyzed using IBM SPSS version 26.0 for Windows (SPSS Inc., Chicago, IL, USA). The normality of the distribution of continuous variables was tested by one-sample Kolmogorov-Smirnov test. Parametric continuous variables were expressed as mean \pm standard deviation, and nonparametric data were shown as median and interquartile ranges. Categorical variables were presented as numbers and proportions. Differences between groups were analyzed by Student $t$ test or Wilcoxon test, as appropriate, for continuous variables, and chi-square test for categorical variables. Paired t-tests were used for monozygotic intrapair comparisons. The differences in abundance of microbial communities between groups were analyzed by Metastat analysis. Pearson or Spearman correlation was respectively used to analyze the correlation between continuous variables with a normal or skewed distribution. $P$ values were adjusted in multiple testing followed by the Benjamini-Hochberg correction. $P<0.05$ was considered significant.

\section{Results}

\section{Intrapair Difference of Clinical with Metabolic Variables of Twins}

For the 12 males and 28 females enrolled in our study, the characteristics of twins according to the level of VAT in each pair are shown in Table 1. The results showed that twins with higher VAT were more likely to have higher values of BMI, body fat percentage and TG (All $P<0.05$, Table 1). There were no statistically significant differences in the concentrations of acetic acid, propionic acid, isobutyric acid, butyric acid, isovaleric acid, valeric acid and caproic acid between the groups $(P>0.05$, Table 1$)$.

\section{Correlation Analysis of SCFAs with Metabolic Parameters}

To further explore the relationship between SCFAs and obesity, we conducted a correlation analysis among all participants. It revealed that propionic acid was negatively correlated with body fat percentage $(r=-0.30, P=$ $0.042)$, waist circumference $(r=-0.42, P=0.008)$, BMI $(r=-0.32, P=0.032)$, and VAT $(r=-0.35, P=0.026)$. A negative relationship was observed between butyric acid and VAT $(r=-0.31, P=0.025)$; isobutyric and isovaleric acid showed negative associations with BMI $(r=-0.32, P=0.037 ; r=-0.38, P=0.046)$ (Figure 1).

\section{Differences in Bacterial Diversity of Gut Microbiota in the Study Group}

In this study, gut microbiota test results from eight volunteers were missing due to insufficient collection of fecal samples; 32 fecal samples were included in the analysis. After data trimming and quality filtering, a data set was generated for $16 \mathrm{~S}$ microbial rDNA sequencing, with an average of 134,779 high-quality sequences per sample (minimum, 80,836; maximum, 399,216). According to VAT of more than $100 \mathrm{~cm}^{2}$ and less than $100 \mathrm{~cm}^{2}$, all subjects were 
Table I Clinical and Biochemical Characteristics of the Subjects

\begin{tabular}{|c|c|c|c|}
\hline \multirow[t]{2}{*}{ Variables } & \multicolumn{2}{|c|}{ Visceral Adipose Tissue } & \multirow[t]{2}{*}{$P$} \\
\hline & Lower in Twins & Higher in Twins & \\
\hline Number & 20 & 20 & \\
\hline Age(years) & $48.5(36.3,55)$ & $48.5(36.3,55)$ & \\
\hline Males/Females & $6 / 14$ & $6 / 14$ & \\
\hline \multicolumn{4}{|l|}{ Adiposity } \\
\hline VAT $\left(\mathrm{cm}^{2}\right)$ & $104.6 \pm 48.1$ & $118.0 \pm 50.5$ & $<0.00 I^{*}$ \\
\hline BFP (\%) & $31.9 \pm 9.0$ & $34.3 \pm 9.2$ & $<0.00 I^{*}$ \\
\hline BMI $\left(\mathrm{kg} / \mathrm{m}^{2}\right)$ & $25.4 \pm 4.7$ & $25.9 \pm 4.7$ & $0.040 *$ \\
\hline$W C(\mathrm{~cm})$ & $85.0 \pm 9.2$ & $90.4 \pm 9.6$ & 0.074 \\
\hline \multicolumn{4}{|l|}{ Blood chemistry } \\
\hline $\mathrm{HDL}(\mathrm{mmol} / \mathrm{l})$ & $\mathrm{I} .4 \pm 0.4$ & $1.4 \pm 0.3$ & 0.690 \\
\hline LDL (mmol/l) & $3.1 \pm 0.8$ & $3.3 \pm 0.9$ & 0.218 \\
\hline $\mathrm{TC}(\mathrm{mmol} / \mathrm{l})$ & $4.7 \pm 1.2$ & $5.3 \pm 1.1$ & 0.114 \\
\hline TG (mmol/l) & $1.2(0.7,1.6)$ & $1.6(0.9,2.0)$ & $0.025^{*}$ \\
\hline FBG $(\mathrm{mmol} / \mathrm{l})$ & $6.0 \pm 1.9$ & $6.5 \pm 3.5$ & 0.314 \\
\hline HbAlc (\%) & $5.9 \pm 1.34$ & $6.1 \pm 1.6$ & 0.421 \\
\hline HOMA-IR & $2.5(1.5,3.9)$ & $2.6(1.2,4.0)$ & 0.370 \\
\hline FINS (ng/mL) & $9.7(6.0,14.6)$ & $9.9(5.5,14.1)$ & 0.502 \\
\hline \multicolumn{4}{|l|}{ Fecal SCFAs } \\
\hline Acetic acid (ug/g) & $1368.4 \pm 709.6$ & $1372.9 \pm 5 \mid 4.6$ & 0.631 \\
\hline Propionic acid (ug/g) & $544.7(358.7,926.2)$ & $697.9(47|.1| 27 \mid .5)$, & 0.100 \\
\hline Isobutyric acid (ug/g) & $68.4(29.2,92.8)$ & $61.5(18.5,132.2)$ & 0.135 \\
\hline Butyric acid (ug/g) & $825.4(397.6,1925.4)$ & $606.9(423.5,1303.3)$ & $0.88 \mathrm{I}$ \\
\hline Isovaleric acid (ug/g) & $69.9(25.2,98.4)$ & $57.2(12.0,134.0)$ & 0.191 \\
\hline Valeric acid (ug/g) & $33.0(11.0,128.3)$ & $79.14(11.7,2 \mid 4.0)$ & 0.073 \\
\hline Caproic acid (ug/g) & $2.5(0.9,33.4)$ & $3.8(0.9,11.9)$ & 0.794 \\
\hline
\end{tabular}

Notes: Continuous variables with normal distribution were presented as mean \pm standard deviation; non-parametric variables were documented as median (interquartile range). *Indicates a significant $P$ value.

Abbreviations: VAT, visceral adipose tissue; BFP, body fat percentage; BMI, body mass index; WC, waist circumference; FBG, fasting blood glucose; HbAlc, glycosylated hemoglobin; HDL, high-density lipoprotein cholesterol; SCFAs, short-chain fatty acids; FINS, fasting insulin; LDL, low-density lipoprotein cholesterol; TC, total cholesterol; TG, triglyceride; HOMA-IR, homeostatic model assessment of insulin resistance.

divided into two groups, namely group with visceral obesity and non-obese group. Chaol and Shannon indexes were used to assess $\alpha$ diversity and this study found no statistical differences in $\alpha$ diversity between the two groups $(P>0.05$, Figure 2). Furthermore, PCoA based on Bray-Curtis distance showed that there were no distinct heterogeneous structures of bacterial community between groups $(P>0.05$, Figure 3$)$.

\section{Differences in Bacterial Genera of Gut Microbiota According to Study Group}

We then compared the gut microbial composition of fecal samples at genus level between groups with and without visceral obesity. Metastat analysis showed that the top 5 relatively abundant bacterial taxa of two groups were Bacteroides, Collinsella, Eubacterium rectale group, Lachnoclostridiumand Tyzzerella (Figure 4). At genus level, compared with non-obese participants, the abundance of Bacteroides, Collinsella were lower in visceral obese patients $(P<0.05$, Figure 4$)$.

\section{Correlation Analysis of Gut Microbiome with SCFAs}

Given the impact of obesity on the SCFAs and gut microbiota, we analyzed the correlations between SCFAs and the relatively abundant bacterial genera associated with visceral obesity. The results showed that the abundance of 41 gut microbiota species was correlated with the concentration of at least one SCFA $(P<0.05$, Figure 5). Among them, the abundance of Collinsella was positively correlated with acetic acid concentration $(r=0.63, P=0.011$, Figure 5). 


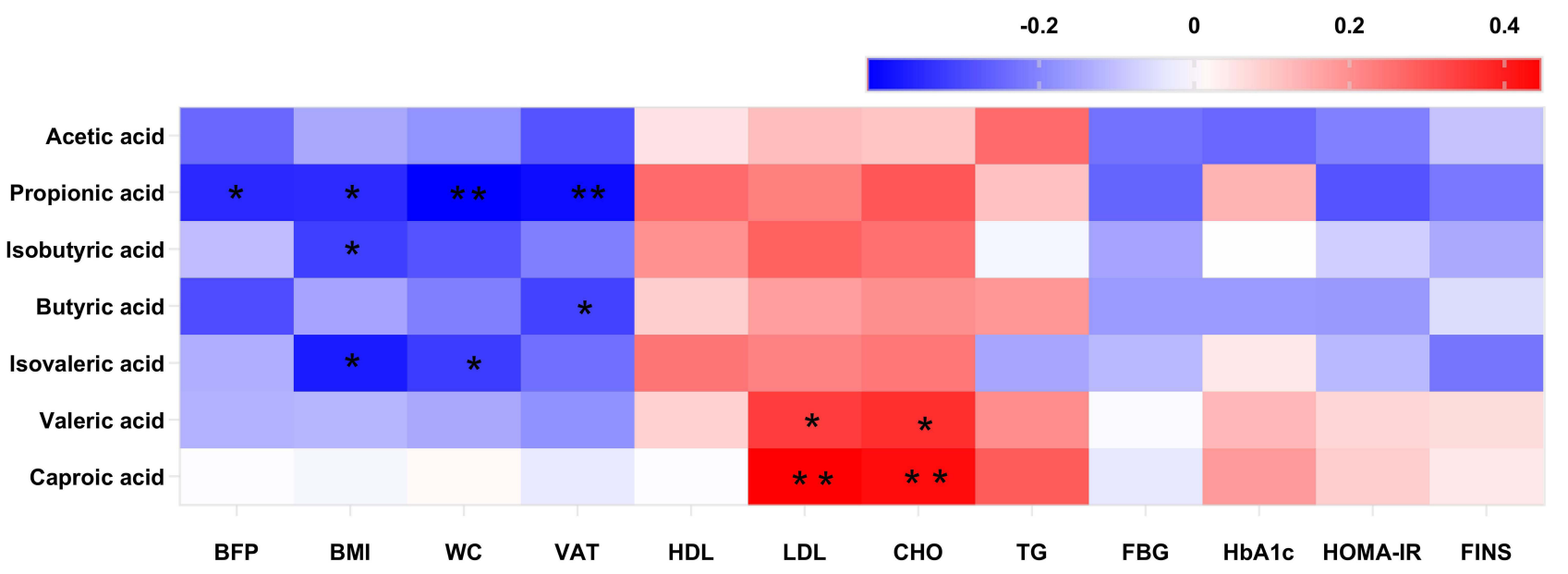

Figure I Correlation analysis of SCFAs, clinical and blood chemistry parameters were displayed in the clustered heatmap. Red is a positive correlation and blue is a negative correlation. The color intensity of the individual rectangles shows the magnitude of the correlation coefficient while the asterisks indicate if the associations are significantly. $* P<0.05 ; * * P<0.01$
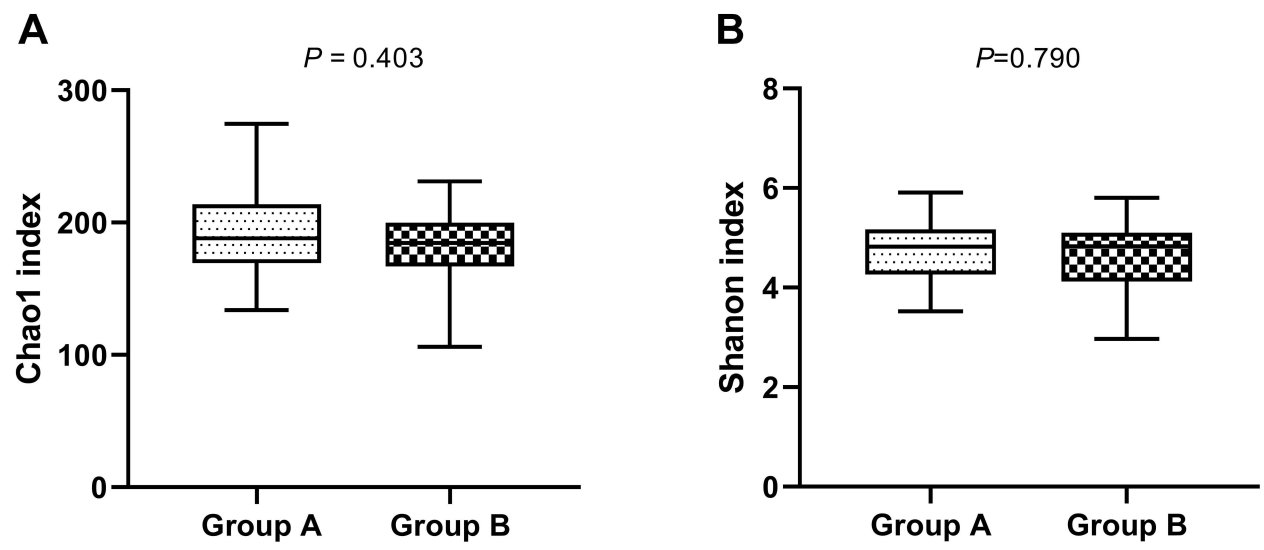

Figure 2 Box-and-whisker plots of $\alpha$ diversity of gut microbiota in fecal samples of participants with visceral obesity (Group A) and non-obesity (Group B). $\alpha$ diversity was estimated using (A) chaol index as an estimate of community richness, (B) Shannon index as an estimate of community diversity.

\section{Discussion}

SCFAs play regulatory functions in the lipids, cholesterol and glucose metabolism, immune response and gut barrier integrity. Our study showed that SCFAs in feces were negatively correlated with adiposity parameters such as BMI, VAT and waist circumference, which is consistent with previous studies. ${ }^{14,15}$ SCFAs have been suggested to mediate the activation of G protein-coupled receptors, such as GPR41 and GPR43, inhibit fat accumulation in adipose tissue and accelerate the metabolism of unincorporated lipids as well as glucose in other tissues, leading to a subsequent improvement in insulin sensitivity. ${ }^{16,17}$ The release of gut-derived satiety hormones like glucagon-like peptide-1 and peptide YY have also been implicated in this action. ${ }^{18,19}$ SCFAs also play a role in the balance of fatty acid synthesis, fatty acid oxidation, and lipolysis in the tissues of the body through peroxisome proliferator-activated receptors. ${ }^{20}$ Additionally, SCFAs were also found to be anti diet-induced obesity. ${ }^{21}$ Dietary supplementation with acetate, propionate, butyrate or 


\section{PC1-PC2}

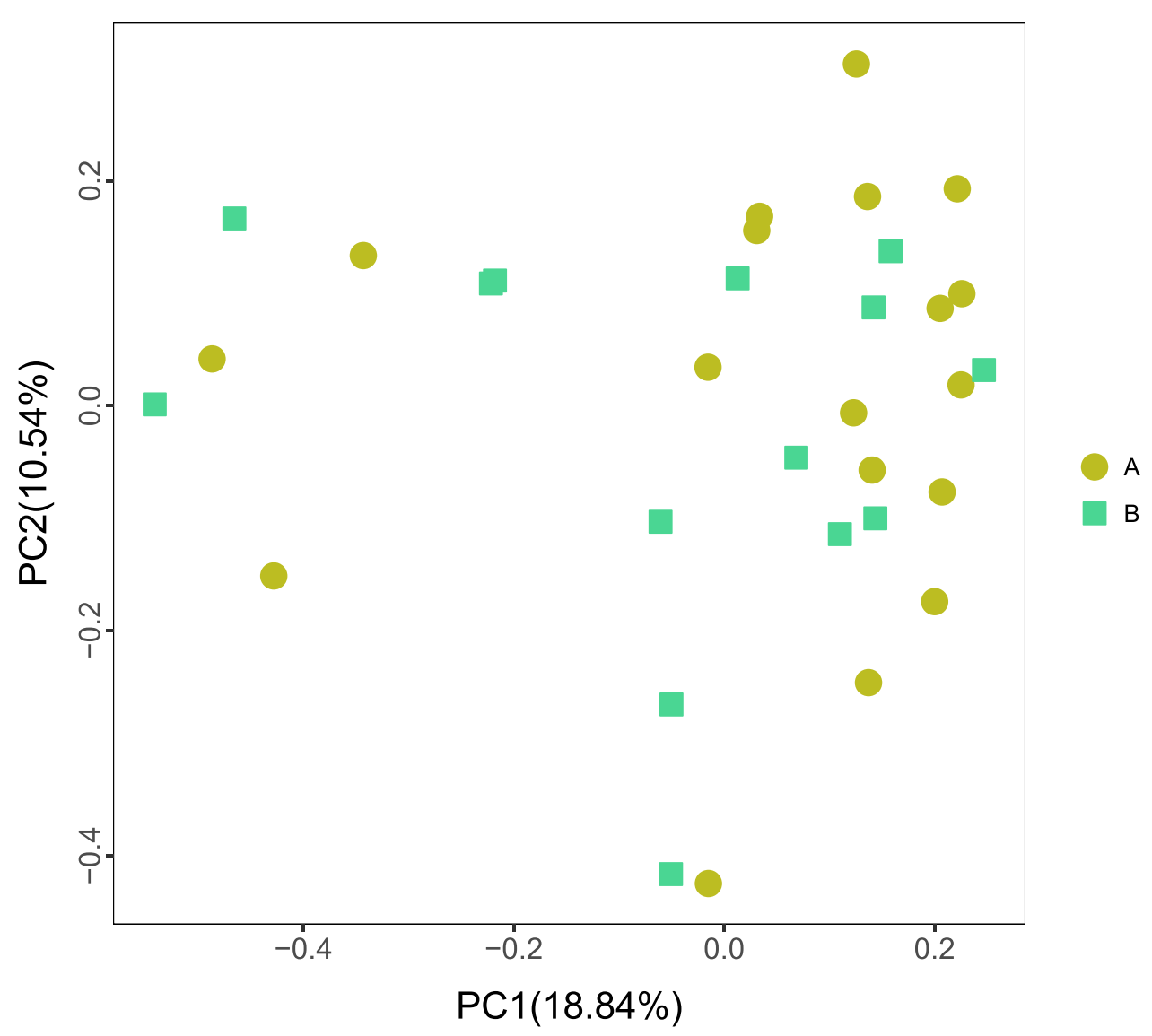

Figure 3 Principal coordinate analysis (PCoA) score plots based on Bray-Curtis distances of fecal microbiota in participants with visceral obesity (Group A) and non-obesity (Group B). $P>0.05$.

their mixture can significantly inhibit the body weight gain induced by high-fat diet feeding. ${ }^{22}$ Our study confirmed the positive effects of SCFAs on obesity.

Recent researches have demonstrated that changes in the gut microbiome are closely linked to metabolic disorders such as obesity and type 2 diabetes, ${ }^{23-25}$ therefore, our study compared the composition of gut microbiota in visceral obese and non-obese participants. Analysis of gut microbiota composition by 16S rRNA gene sequencing revealed that participants with visceral obesity had lower abundance of Bacteroides and Collinsella compared to non-obese patients. Previous studies have shown that in subjects with obesity, intestinal dysbiosis appears to be related to the abundance of Bacteroides and the species Eubacterium rectale. ${ }^{26}$ Collinsella has been shown to be associated with triglycerides and cholesterol, ${ }^{27}$ the gut microbial diversity is reconstituted and the relative abundance of Collinsella is increased in obese mice after bariatric surgery. ${ }^{28}$ In terms of diet, whole grain diet and solventable dietary fiber may enable the overgrowth of Collinsella. ${ }^{29,30}$ The treatment with human origin-probiotics has been shown to ameliorate gut microbiome dysbiosis by enhancing SCFAs production. ${ }^{31,32}$ Our study also showed a positive association between Collinsella and concentrations of acetic acid, suggesting that Collinsella may influence VAT by regulating SCFAs. Similar to our study, several previous studies have confirmed the correlation between SCFAs and gut microbiota such as Eubacterium_coprostanoligenes_group and Roseburia, therefore, we 

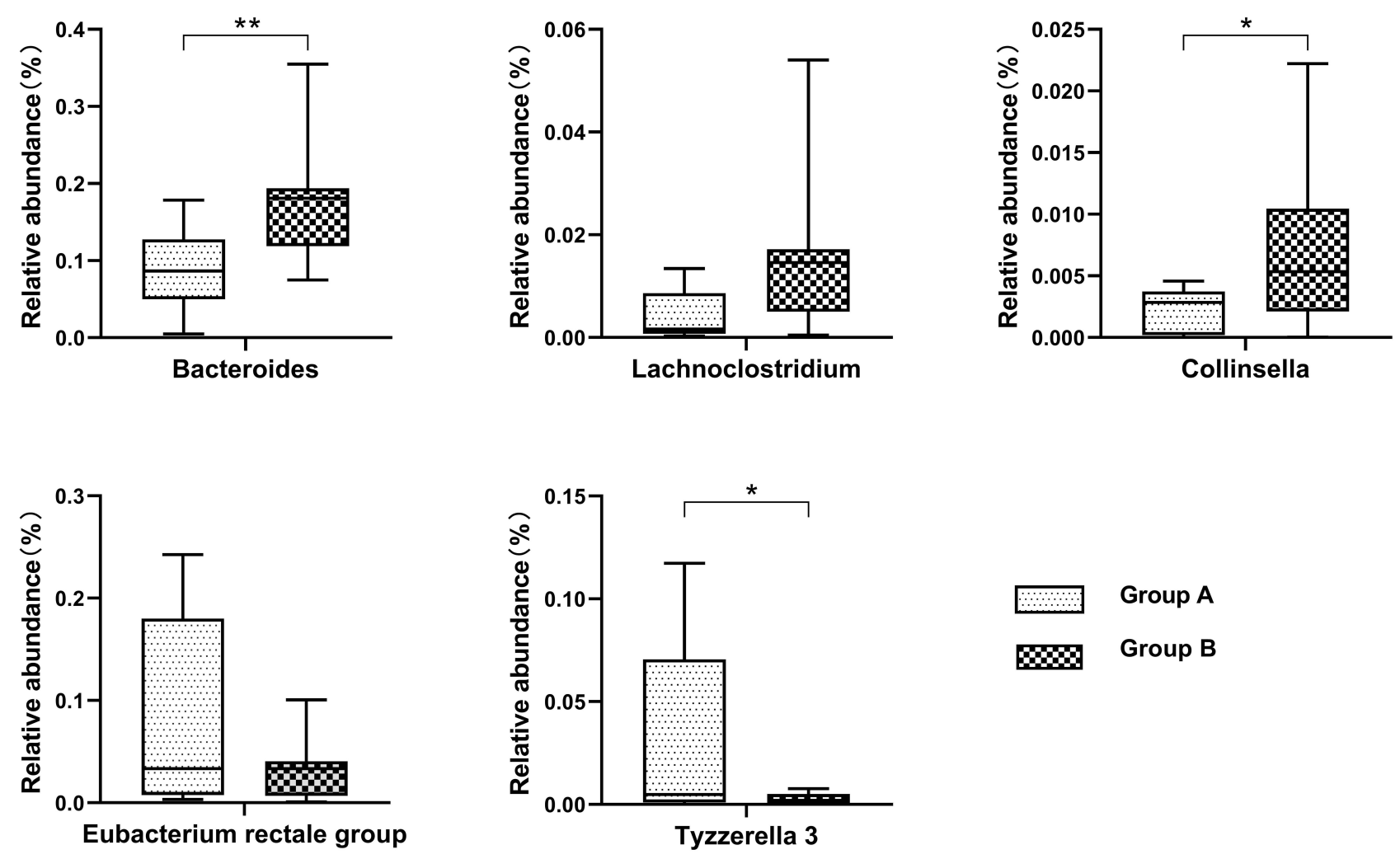

Figure 4 Box-and-whisker plots illustrating the differences of the top 5 bacterial relative abundances in participants with visceral obesity (Group A) and non-obesity (Group B). The median and $95 \%$ confidence intervals are shown. $* P<0.05 ; * * P<0.01$.

speculate that the mechanism by which the gut microbiome affects host physiology may be partly mediated by SCFAs.

Monozygotic twins share $100 \%$ of their genomic sequence and are perfectly or closely matched for many environmental factors. Using monozygotic twins as subjects can explore the potential modificatory effects of obesity on the body under controlled genetic and shared familial environmental factors. Unfortunately, no statistically significant intrapair differences were observed in SCFAs between monozygotic twins in this study. In previous studies, the heritability estimates for VAT range from $42 \%$ to $57 \% .{ }^{33,34}$ Similarly, the average intrapair difference in VAT of MZ twins was only $14 \mathrm{~cm}^{2}$ in this study, which may not be enough to cause significant difference in short chain fatty acid concentration.

The major strength in our study is that we performed in MZ twins, who shared the same genetic background and grew up in a similar environment, reducing confounding factors in the study. However, there were also several limitations in our study. First, the sample size in the study was relatively small. Second, SCFAs were detected only in feces, not in the serum. Our team has already performed the analysis of SCFAs in serum, and more valuable information is expected from the analysis.

\section{Conclusion}

Low fecal concentrations of short-chain fatty acid were associated with visceral obesity, and the gut microbiota might be involved in the underlying mechanism.

\section{Author Contributions}

Xing-Qi Yin and Ya-Xin An contributed equally to this study and should be considered co-first authors for this study. Ke Yu designed and conducted the study. Xing-Qi Yin and Ya-Xin An analyzed the data and drafted the manuscript. Cai-Guo 


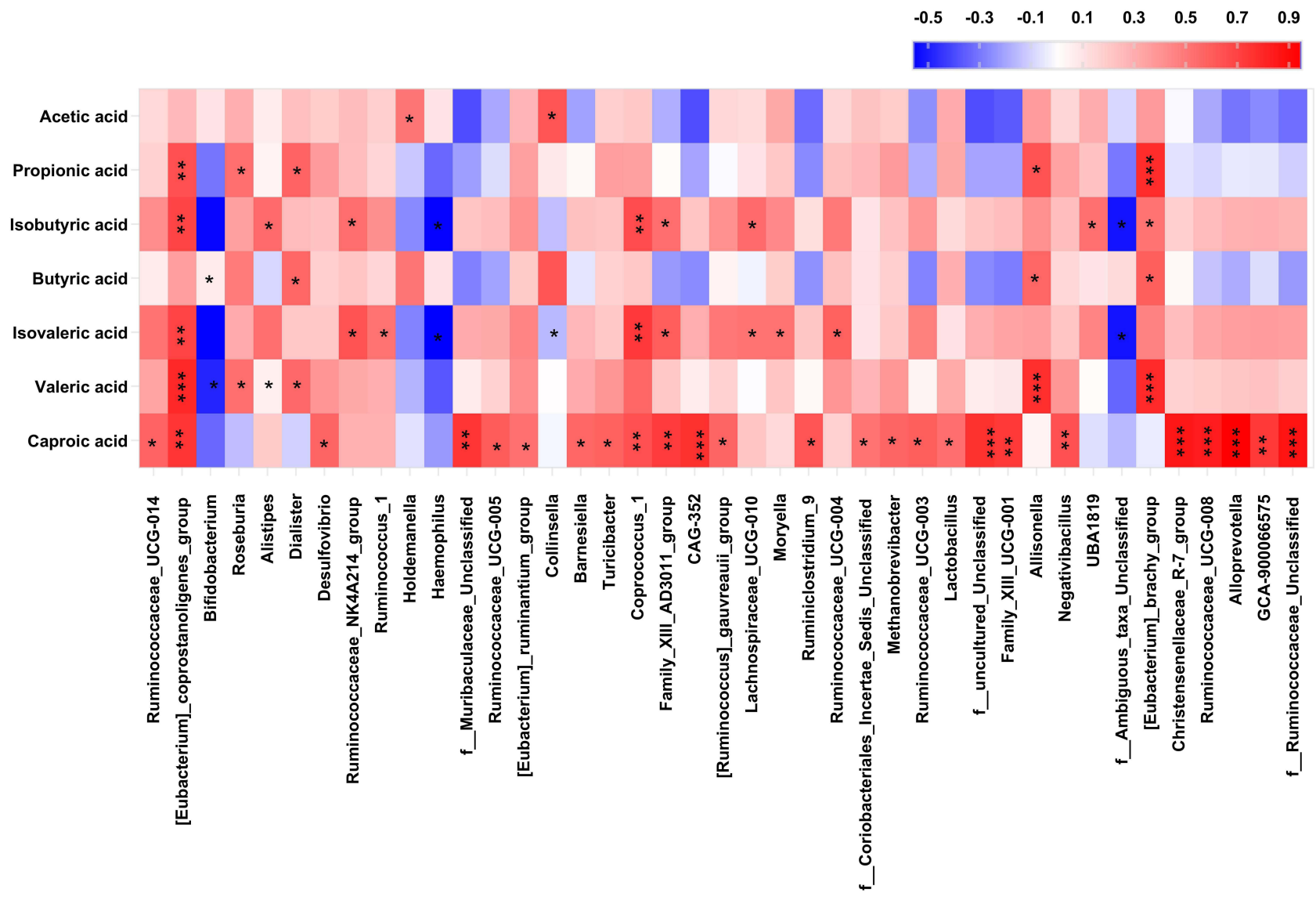

Figure 5 Heatmap displaying Spearman correlation coefficients between bacterial taxa and fecal SCFAs. Red represents a positive correlation and blue indicates a negative correlation. The color intensity of the individual rectangles shows the magnitude of the correlation coefficient while the asterisks indicate if the associations are significantly. $* 0.01<P<0.05 ; * * 0.001<P<0.01 ; * * * P<0.001$.

Yu, Jing Ke and Dong Zhao reviewed the manuscript. All authors made substantial contributions to conception and design, acquisition of data, or analysis and interpretation of data; took part in drafting the article or revising it critically for important intellectual content; agreed to submit to the current journal; gave final approval of the version to be published; and agree to be accountable for all aspects of the work.

\section{Disclosure}

The authors report no conflicts of interest in this work.

\section{References}

1. Engin A. The definition and prevalence of obesity and metabolic syndrome. Adv Exp Med Biol. 2017;960:1-17. doi:10.1007/978-3-319-48382-5_1

2. Bastien M, Poirier P, Lemieux I, Despres JP. Overview of epidemiology and contribution of obesity to cardiovascular disease. Prog Cardiovasc Dis. 2014;56(4):369-381. doi:10.1016/j.pcad.2013.10.016

3. Hooper LV, Gordon JI. Commensal host-bacterial relationships in the gut. Science. 2001;292(5519):1115-1118. doi:10.1126/science.1058709

4. Gomes AC, Hoffmann C, Mota JF. The human gut microbiota: metabolism and perspective in obesity. Gut Microbes. 2018;9:308-325. doi:10.1080/ 19490976.2018.1465157

5. Rao Y, Kuang Z, Li C, et al. Gut Akkermansia muciniphila ameliorates metabolic dysfunction-associated fatty liver disease by regulating the metabolism of L-aspartate via gut-liver axis. Gut Microbes. 2021;13:1-19. doi:10.1080/19490976.2021.1927633

6. Fan Y, Pedersen O. Gut microbiota in human metabolic health and disease. Nat Rev Microbiol. 2021;19:55-71. doi:10.1038/s41579-020-0433-9

7. Byrne CS, Chambers ES, Morrison DJ, Frost G. The role of short chain fatty acids in appetite regulation and energy homeostasis. Int J Obes (Lond). 2015;39:1331-1338. doi:10.1038/ijo.2015.84

8. Barron R, Bermingham K, Brennan L, et al. Twin metabolomics: the key to unlocking complex phenotypes in nutrition research. Nutr Res. 2016;36:291-304. doi:10.1016/j.nutres.2016.01.010 
9. van Dongen J, Slagboom PE, Draisma HH, Martin NG, Boomsma DI. The continuing value of twin studies in the omics era. Nat Rev Genet. 2012;13(9):640-653. doi:10.1038/nrg3243

10. Song YM, Sung J, Lee K. Associations between adiposity and metabolic syndrome over time: the healthy twin study. Metab Syndr Relat Disord. 2017;15(3):124-129. doi:10.1089/met.2016.0100

11. Bunning BJ, Contrepois K, Lee-McMullen B, et al. Global metabolic profiling to model biological processes of aging in twins. Aging Cell. 2020;19: e13073. doi:10.1111/acel.13073

12. Ramo JT, Kaye SM, Jukarainen S, et al. Liver fat and insulin sensitivity define metabolite profiles during a glucose tolerance test in young adult twins. J Clin Endocrinol Metab. 2017;102(1):220-231. doi:10.1210/jc.2015-3512

13. Jukarainen S, Holst R, Dalgård C, et al. Cardiorespiratory fitness and adiposity as determinants of metabolic health-pooled analysis of two twin cohorts. J Clin Endocrinol Metab. 2017;102(5):1520-1528. doi:10.1210/jc.2016-3435

14. Miranda VPN, Dos Santos Amorim PR, Bastos RR, et al. Abundance of gut microbiota, concentration of short-chain fatty acids, and inflammatory markers associated with elevated body fat, overweight, and obesity in female adolescents. Mediators Inflamm. 2019;2019:7346863. doi:10.1155/ 2019/7346863

15. Murugesan S, Ulloa-Martínez M, Martínez-Rojano H, et al. Study of the diversity and short-chain fatty acids production by the bacterial community in overweight and obese Mexican children. Eur J Clin Microbiol Infect Dis. 2015;34(7):1337-1346. doi:10.1007/s10096-015-2355-4

16. Shimizu H, Masujima Y, Ushiroda C, et al. Dietary short-chain fatty acid intake improves the hepatic metabolic condition via FFAR3. Sci Rep. 2019;9(1):16574. doi:10.1038/s41598-019-53242-x

17. Ang Z, Ding JL. GPR41 and GPR43 in obesity and inflammation - protective or causative? Front Immunol. 2016;7:28. doi:10.3389/ fimmu.2016.00028

18. Hernandez MAG, Canfora EE, Jocken JWE, Blaak EE. The short-chain fatty acid acetate in body weight control and insulin sensitivity. Nutrients. 2019;11(8):1943. doi:10.3390/nu11081943

19. Larraufie P, Martin-Gallausiaux C, Lapaque N, et al. SCFAs strongly stimulate PYY production in human enteroEndocrine cells. Sci Rep. 2018;8:74. doi:10.1038/s41598-017-18259-0

20. Strickland JC, Stoops WW. Perceptions of research risk and undue influence: implications for ethics of research conducted with cocaine users. Drug Alcohol Depend. 2015;156:304-310. doi:10.1016/j.drugalcdep.2015.09.029

21. Matey-Hernandez ML, Williams FMK, Potter T, et al. Genetic and microbiome influence on lipid metabolism and dyslipidemia. Physiol Genomics. 2018;50:117-126. doi:10.1152/physiolgenomics.00053.2017

22. Ellis MS, Cicero TJ, Dart RC, Green JL. Understanding multi-pill ingestion of prescription opioids: prevalence, characteristics, and motivation. Pharmacoepidemiol Drug Saf. 2019;28(1):117-121. doi:10.1002/pds.4687

23. Zhi C, Huang J, Wang J, et al. Connection between gut microbiome and the development of obesity. Eur J Clin Microbiol Infect Dis. 2019;38:1987-1998. doi:10.1007/s10096-019-03623-x

24. Lee CJ, Sears CL, Maruthur N. Gut microbiome and its role in obesity and insulin resistance. Ann N Y Acad Sci. 2020;1461:37-52. doi:10.1111/ nyas. 14107

25. Vangipurapu J, Fernandes Silva L, Kuulasmaa T, Smith U, Laakso LM. Microbiota-related metabolites and the risk of Type 2 diabetes. Diabetes Care. 2020;43(6):1319-1325. doi:10.2337/dc19-2533

26. Yang JY, Lee Y-S, Kim Y, et al. Gut commensal Bacteroides acidifaciens prevents obesity and improves insulin sensitivity in mice. Mucosal Immunol. 2017;10:104-116. doi:10.1038/mi.2016.42

27. Astbury S, Atallah E, Vijay A, et al. Lower gut microbiome diversity and higher abundance of proinflammatory genus Collinsella are associated with biopsy-proven nonalcoholic steatohepatitis. Gut Microbes. 2020;11:569-580. doi:10.1080/19490976.2019.1681861

28. Frost F, Storck LJ, Kacprowski T, et al. A structured weight loss program increases gut microbiota phylogenetic diversity and reduces levels of Collinsella in obese type 2 diabetics: a pilot study. PLoS One. 2019;14:e0219489. doi:10.1371/journal.pone.0219489

29. Foerster J, Maskarinec G, Reichardt N, et al. The influence of whole grain products and red meat on intestinal microbiota composition in normal weight adults: a randomized crossover intervention trial. PLoS One. 2014;9:e109606. doi:10.1371/journal.pone.0109606

30. Reimer RA, Wharton S, Green TJ, et al. Effect of a functional fibre supplement on glycemic control when added to a year-long medically supervised weight management program in adults with type 2 diabetes. Eur J Nutr. 2021;60:1237-1251. doi:10.1007/s00394-020-02328-8

31. Schmidt TSB, Raes J, Bork P. The human gut microbiome: from association to modulation. Cell. 2018;172:1198-1215. doi:10.1016/j. cell.2018.02.044

32. Nagpal R, Wang S, Ahmadi S, et al. Human-origin probiotic cocktail increases short-chain fatty acid production via modulation of mice and human gut microbiome. Sci Rep. 2018;8:12649. doi:10.1038/s41598-018-30114-4

33. Hong Y, Rice T, Gagnon J, et al. Familial clustering of insulin and abdominal visceral fat: the HERITAGE family study. J Clin Endocrinol Metab. 1998;83:4239-4245. doi:10.1210/jcem.83.12.5312

34. Perusse L, Després JP, Lemieux S, et al. Familial aggregation of abdominal visceral fat level: results from the Quebec family study. Metabolism. 1996;45:378-382. doi:10.1016/s0026-0495(96)90294-2 


\section{Publish your work in this journal}

Diabetes, Metabolic Syndrome and Obesity: Targets and Therapy is an international, peer-reviewed open-access journal committed to the rapid publication of the latest laboratory and clinical findings in the fields of diabetes, metabolic syndrome and obesity research. Original research, review, case reports, hypothesis formation, expert opinion and commentaries are all considered for publication. The manuscript management system is completely online and includes a very quick and fair peer-review system, which is all easy to use. Visit http://www.dovepress. com/testimonials.php to read real quotes from published authors.

Submit your manuscript here: https://www.dovepress.com/diabetes-metabolic-syndrome-and-obesity-targets-and-therapy-journal 